\title{
POURQUOI FRANÇAIS EN FACE DE FRANÇOIS?
}

Le lat. -ensem et le germ. -isk se sont confondus en français en un seul suffixe qui apparaît sous une double forme, -ois et -ais. Spore (1989: 358-359) est le dernier auteur à se demander de quoi dépend la répartition -ais/-ois:

“-ais et -ois, d'origine commune, se font concurrence. Plattner voulait déterminer la répartition d'après des critères phonétiques, mais Collijn s'y est opposé violemment en misant sur le critère géographique: -ais à l'ouest, -ois à l'est.

De nos jours, Heinz Jürgen Wolf se joint au point de vue de Collijn, alors que Jean-Claude Dinguirard se fait le porte-parole du critère phonétique.

A partir d'un inventaire de 2114 noms (901 prenant -ais, 1213 -ois), je constate qu'un critère comme l'autre est insuffisant, car même là où un des deux suffixes domine très nettement, il y a des exceptions. Cela est vrai aussi bien pour le critère géographique (aucun département ne montre un suffixe à $100 \%$ ) que pour le critère phonétique (aucun contexte phonétique ne provoque tel suffixe dans tous les cas).

Après avoir étudié rapidement les suffixes que affectent les noms terminés en -ange, -ville et $-a c$, j'esquisse une théorie selon laquelle il y aurait interdépendance entre les deux critères, si bien qu'un nom qui, phonétiquement, devrait prendre -ois, peut prendre -ais dans les zones où ce suffixe domine, de même qu'un nom placé dans une aire à -ois, peut prendre -ais pour des motifs phonétiques. Il pourrait aussi y avoir des contextes phonétiques et des zones géographiques neutres devant le choix du suffixe.

Tout cela dépend en quelque sorte de la loi du plus fort, c'est-à-dire du critère qui, dans chaque cas particulier, a le plus de poids. Déterminer le poids de ces critères, voilà un énorme travail qui reste encore à faire.“

Bien des questions se posent. Pourquoi l'auteur examine-t-il les adjectifs tirés de noms de ville de France et fait-il abstraction de ceux s'appliquant à l'étranger, tel munichois? Pourquoi ne tient-il pas compte des adjectifs tirés de noms de provinces ou de pays, tels dauphinois ou portugais? Pourquoi ne s'occupe-t-il pas des substantifs, tel Néerlandais? Pourquoi ne prend-il pas en considération les prénoms, tel François? Pourquoi ne s'intéresse-t-il pas aux appellatifs, tel bourgeois? 
A notre avis, il faut considérer tous les dérivés en -ois et -ais et, en tenant compte de tous les dérivés en question, nous sommes arrivé, il y a déjà longtemps, à la conclusion que le lat. -ensem et le germ. -isk ont abouti régulièrement à -ois, tandis que -ais s'explique par ce que nous appelons un développement phonétique irrégulier dû à la fréquence d'emploi, voir Mańczak 1962: 32. Nous avons répété cette opinion à différentes occasions, cf. Mańczak 1969: 69-70, 1974 ou bien 1987: 115.

Résumée au maximum, la théorie du développement phonétique irrégulier dû à la fréquence d'emploi se présente comme suit. Une loi synchronique précise que les éléments linguistiques plus employés sont plus petits que ceux usités moins fréquemment. Il existe une sorte d'équilibre entre le volume des éléments linguistiques et leur fréquence. Mais le volume des éléments linguistiques n'est pas stable. Par suite du développement phonétique régulier, la longueur des mots peut changer sensiblement, comme le montre la comparaison de quelques mots latins et français:

$\begin{array}{lll}\text { me }(2 \text { phonèmes) } & \text { moi }(3 \text { phonèmes) } & \text { augmentation de } 50 \% \\ \text { rem }(3) & \text { rien }(3) & \text { aucun changement } \\ \text { bene }(4) & \text { bien }(3) & \text { diminution de } 25 \% \\ \text { amicam }(6) & \text { amie }(3) & \text { diminution de } 50 \% \\ \text { bellos }(6) & \text { beaux }(2) & \text { diminution de } 67 \% \\ \text { Augustum }(8) & \text { août }(1) & \text { diminution de } 88 \%\end{array}$

La fréquence des mots n'est pas stable non plus, par exemple sire est moins employé de nos jours qu'au moyen âge, tandis que chauffeur est plus utilisé maintenant que dans le passé. Dans cet état de choses, il peut se produire que l'équilibre entre volume et fréquence soit bouleversé. Si un élément linguistique devient trop court par rapport à sa fréquence, on l'allonge, cf. août $[\mathrm{u}]$ remplacé par [ut] ou mois d'août. Si, au contraire, un élément linguistique devient trop long par rapport à sa fréquence, il est nécessaire que l'équilibre soit rétabli par la diminution de son volume. Et l'on sait qu'il y a des abrégements provoqués par l'augmentation de la fréquence d'emploi, qu'un groupe de mots peut être réduit à un seul mot (chemin de fer métropolitain>métropolitain), qu'un mot composé peut être réduit à un seul élément (télévision>télé), que n'importe quel mot peut être amputé d'une partie de son corps (faculté> fac), mais les linguistes ont de la peine à comprendre que, à côté de ces abrégements pour ainsi dire "mécaniques", il y en ait d'autres qui se produisent dans les radicaux ( $\left.{ }^{*} a v r-a i>a u r-a i\right)$, les affixes (prem-ier, où [r] est tombé) et les désinences (cant-avit>chant-a). Alors que les plus grands romanistes du XIX ${ }^{\mathrm{e}}$ siècle, tels Diez (1846: 12) ou Schuchardt (1885), commençaient à entrevoir cette vérité si simple, dans la $3^{\mathrm{e}}$ édition du dictionnaire étymologique de Bloch et Wartburg, il n'y a qu'un seul mot, la conjonction que, dont il est dit que "son fréquent usage l'affaiblit en que“.

La question se pose, de savoir comment on peut reconnaitre qu'une irrégularité du développement phonétique s'explique par la fréquence. Les irrégularités de ce type se caractérisent par deux traits: 
a) Elles consistent toujours dans une réduction irrégulière du morphème, mot ou groupe de mots. Si le développement phonétique irrégulier dû à la fréquence est assez avancé, il consiste en la chute d'un ou de plusieurs phonèmes, par exemple

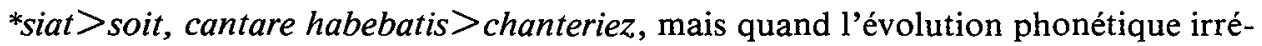
gulière se trouve dans sa phase initiale, elle ne consiste que dans une réduction partielle dụ phonème. Voici quelques exemples:

la quantité de la voyelle peut être réduite, $\mathrm{cf}$. nostrum $>$ nôtre $>$ notre;

le timbre de la voyelle peut être réduit, cf. le futur fera de faire en regard du futur régulier plaira de plaire;

l'aperture de la voyelle peut être réduite $(a>o>u$ ou bien $a>e>i)$, cf. cantamus $>$ chantons, a. esp. sen $>\sin$;

une consonne palatale ( = consonne non mouillée + palatalité) peut être réduite en une consonne non palatale, par ex. illos est devenu en espagnol los, bien que le $l$ mouillé existe en espagnol à l'initiale, cf. llano.

b) Les changements phonétiques irréguliers en question n'ont lieu que dans les morphèmes, mots ou groupes de mots très employés. Il y a six moyens de reconnaitre qu'il s'agit en effet de tels éléments linguistiques:

$1^{\circ}$ S'il existe, pour la langue et la période en question, un dictionnaire de fréquences, on peut, évidemment, l'utiliser parce que la grande majorité des mots subissant un développement phonétique irrégulier dû à la fréquence se trouvent parmi les mille mots les plus employés dans la langue en question.

$2^{\circ}$ Si le morphème, mot ou groupe de mots apparaît dans une langue donnée sous une double forme, régulière et irrégulière, le développement phonétique irrégulier dû à la fréquence se caractérise par le fait que la forme irrégulière est, en général, plus employée que la forme régulière, par exemple aller est plus employé que ambler et il en est de même pour monsieur et monseigneur.

$3^{\circ}$ Si les changements phonétiques irréguliers dus à la fréquence se produisent à l'intérieur d'un paradigme flexionnel ou d'une famille de mots, les réductions ont lieu plus souvent dans les formes plus fréquentes que dans les formes plus rares. Par ex., parmi les formes italiennes ho, hai, ha, abbiamo, avete, hanno sont irréguliers ho, hai, ha, hanno, ce qui s'explique par le fait que le singulier est plus employé que le pluriel, et que la troisième personne est plus utilisée que les autres. La différence entre le consonantisme initial de l'all. sollen et celui de Schuld, schuldig s'explique par le fait que le verbe en question est plus employé que le nom et l'adjectif.

$4^{\circ} \mathrm{A}$ côté du développement phonétique irrégulier dû à la fréquence, il existe une autre évolution irrégulière, qui consiste en des accidents phonétiques connus de- 
puis longtemps sous le nom d'assimilations, dissimilations ou métathèses, en formes hypercorrectes ou formes expressives. Toute cette évolution irrégulière se caractérise par le fait qu'elle a lieu, dans différentes langues, dans des mots divers. Le mot français chercher < cercher présente une assimilation, faible < flebilem une dissimilation, troubler<turbulare une métathèse, tandis que $h$ dans herse est expressif. Mais il serait difficile de trouver, dans une langue indo-européenne, un mot signifiant "chercher" qui présenterait une assimilation, un mot signifiant "faible" avec une dissimilation, un mot signifiant "troubler" avec une métathèse ou bien un mot signifiant "herse" avec un phonème d'origine expressive. Bref, il n'y a aucun parallélisme entre les irrégularités dites assimilations, dissimilations, métathèses, etc., qui se produisent dans des langues différentes. Au contraire des assimilations, des dissimilations, etc., le développement phonétique irrégulier dû à la fréquence a lieu, dans diverses langues, d'une manière plus ou moins parallèle, ce qui s'explique par le fait que, malgré les différences qui séparent les communautés linguistiques, les mots les plus fréquents sont partout plus ou moins les mêmes. Par exemple, le verbe signifiant "parler" présente un développement irrégulier dans beaucoup de langues, cf. fr. parler et it. parlare<parabolare, lat. ajo <*agio (en face du développement régulier dans les substantifs adagio ou prodigium, qui sont moins employés), roum. vorbi $<d v o r b i$, sarde nau $<$ narro, nas $<$ narras, etc., angl. says, said (en face du régulier lays, laid), pol. mówić $<$ motwić ou bien russe dial. gyt $<$ gryt $<$ govorit.

$5^{\circ}$ Quand on a à sa disposition un dictionnaire de fréquences et un dictionnaire inverse, on peut examiner des séries de mots qui commencent ou se terminent par la même lettre ou les mêmes lettres. Il est significatif par exemple qu'en anglais, parmi les mots terminés en -iness, business soit le seul mot dont la prononciation est réduite et que ce mot soit plus employé que tous les autres derivés en -iness. Parmi les mots qui ont autrefois commencé par $h$-, l'anglais it $(<h i t)$ est le seul mot qui, dans l'orthographe officielle, ait perdu la consonne initiale; en même temps, il est plus usité que tous les mots qui ont gardé $h$-. Évidemment, cela prouve que les changements business $>$ [biznis] et hit $>$ it s'expliquent par un développement phonétique irrégulier dû à la fréquence.

$6^{\circ}$ Quand on a à sa disposition un atlas linguistique, on peut observer que de deux (ou plusieurs) changements s'expliquant par un développement phonétique irrégulier dû à la fréquence, celui qui est le plus fréquent a une aire plus grande que l'autre (ou les autres). En français le $r$ final se maintient (aurum $>$ or, florem $>$ fleur, etc.), mais beaucoup d'infinitifs présentent une chute irrégulière du $-r$. L'examen de cartes de l' $A L F$ montre que la chute de $-r$ est la plus répandue dans les verbes en -er, moins répandue dans les verbes en -ir et la moins répandue dans les verbes en -oir. Ceci s'explique par le fait que les verbes en -er sont les plus fréquents, ceux en -ir sont moins employés et ceux en -oir, les moins employés.

En ce qui concerne la question de savoir pourquoi le lat. -ensem et le germ. -isk se sont confondus en français en un seul suffixe qui apparaît sous une double forme, -ois et -ais, nous estimons que -ois, prononcé autrefois [w $\varepsilon$ ], tantôt a abouti réguliè- 
rement à [wa], tantôt s'est réduit en $[\varepsilon]$, en subissant un développement phonétique irrégulier dû à la fréquence. Nous avons dépouillé Le Monde du 8 et du 21 septembre 1989 , où nous avons trouvé les dérivés suivants en -ois et en -ais (les nombres indiquent la fréquence):

-ois: 13 chinois; 10 François; 2 québécois, suédois; 1 berlinois, dauphinois, hongrois, Luxembourgeois, munichois, patois, Rueillois.

-ais: 112 français; 23 polonais; 22 anglais; 11 japonais; 10 libanais; 6 néerlandais; 3 hollandais, lyonnais, portugais; 2 new-yorkais; 1 aveyronnais, bourbonnais, écossais, irlandais, pakistanais.

Au point de vue statistique, ces données se présentent comme suit:

$\begin{array}{cccc} & \begin{array}{c}\text { Nombre } \\ \text { d'attestations }\end{array} & \begin{array}{c}\text { Nombre } \\ \text { de mots }\end{array} & \begin{array}{c}\text { Fréquence d'emploi } \\ \text { moyenne }\end{array} \\ \text {-ois } & 34 & 11 & 3 \\ \text {-ais } & 200 & 15 & 13\end{array}$

Il en résulte que la fréquence d'emploi moyenne des dérivés en -ois (à developpement phonétique régulier) a été basse, tandis que celle des dérivés en -ais (à développement phonétique irrégulier) a été haute. Autrement dit, il y a un rapport de cause à effet entre la réduction du suffixe -ois [w $[$ ] en -ais [ $\varepsilon]$ et la haute fréquence d'emploi. C'est dire que, en ce qui concerne la question posée dans le titre du présent article, la réponse est la suivante: le suffixe s'est développé dans François normalement parce que ce prénom était peu employé, et a subi une réduction dans Français parce que cet ethnonyme était très employé. Autrement dit, le rapport entre Français et François est comparable à celui qu'il y a entre des mots comme aller ou monsieur (très employés) et des mots comme ambler ou monseigneur (peu employés).

A l'appui de cette thèse, on peut citer encore des données relatives au développement du suffixe -isk en germanique parce que, nous l'avons dit, le développement phonétique irrégulier dû à la fréquence se caractérise entre autres par le fait qu'il se produit souvent d'une façon plus ou moins parallèle dans différentes langues. $\mathrm{Vu}$ que le suffixe français -ois/-ais présente une certaine ressemblance sémantique (et même étymologique) avec le suffixe germanique -isk, il est instructif d'examiner comment ce dernier s'est développé en suédois, en allemand et en néerlandais.

En ce qui concerne le suédois, nous avons dépouillé un fragment du journal $D a$ gens $N y$ heter du 5 décembre 1981 , où nous avons trouvé les formes suivantes en -isk et $-s k$ :

-isk: 13 politisk; 7 ekonomisk, nordisk; 6 brittisk, symmetrisk; 4 faktisk, praktisk, saudiarabisk; 3 demokratisk, psykisk, skandinavisk, socialdemokratisk; 2 ara- 
bisk, babylonisk, dramatisk, europeisk, källkritisk, mekanisk, österrikisk, saudisk; 1 aktivistisk, automatisk, belgisk, byråkratisk, diplomatisk, elektronisk, filosofisk, heletronisk, juridisk, kanadensisk, kommunistisk, kritisk, kronisk, legendarisk, metafysisk, moralisk, obligatorisk, ökonomisk, okritisk, opartisk, planekonomisk, profetisk, psykologisk, realistisk, retorisk, socialistisk, sovjetisk, spontanistisk, statistisk, stereometrisk, systematisk, taktisk, teknisk, tjeckisk, typisk, utopisk, vegetarisk.

-sk: 35 svensk; 30 dansk; 11 amerikansk, fransk; 5 afrikansk, tysk; 4 engelsk, norsk; 2 japansk, rysk, västtysk; 1 holländsk, inhemsk, medicinsk, platonsk, polsk, skånsk, stockholmsk, utländsk, västerländsk.

Au point de vue statistique, ces données se présentent comme suit:

$\begin{array}{lccc} & \begin{array}{c}\text { Nombre } \\ \text { d'attestations }\end{array} & \begin{array}{c}\text { Nombre } \\ \text { de mots }\end{array} & \begin{array}{c}\text { Fréquence d'emploi } \\ \text { moyenne }\end{array} \\ -i s k & 116 & 57 & 2 \\ -s k & 120 & 20 & 6\end{array}$

Il en résulte que la fréquence d'emploi moyenne des dérivés en - $s k$ (à développement phonétique irrégulier) est plus élevée que celle des dérivés en -isk (à développement phonétique régulier).

En ce qui concerne l'allemand, nous avons dépouillé le journal Berliner Zeitung am Abend du 10 octobre 1989, où nous avons trouvé les formes suivantes en -isch et -sch:

-isch: 21 sowjetisch; 12 sozialistisch; 7 (nordwest)europäisch; 5 (außen)politisch, musikalisch, (nordost)französisch; 3 (anti)faschistisch, johannisch; 2 demokratisch, humanistisch, kritisch, künstlerisch, literarisch, märkisch, militärisch, polnisch, russisch, sängerisch, telefonisch; 1 afroamerikanisch, argentinisch, ausländisch, authentisch, bayerisch, bengalisch, bulgarisch, chemisch, chinesisch, despotisch, drastisch, elektronisch, euphorisch, extremistisch, faktisch, feuilletonistisch, griechisch, islamisch, italienisch, jemenitisch, kommunistisch, lakonisch, magisch, malerisch, medizinisch, moslemisch, nichtarisch, niederländisch, niedersächsisch, nikaraguanisch, ökonomisch, palästinensisch, parlamentarisch, philharmonisch, poetisch, praktisch, proletarisch, rassistisch, rumänisch, schöpferisch, schriftstellerisch, schwärmerisch, schweizerisch, seelisch, spanisch, sporadisch, südafrikanisch, tierisch, tschechoslowakisch, türkisch, ungarisch.

-sch: 63 (West)deutsch(land).

Au point de vue statistique, ces données se présentent comme suit: 


$\begin{array}{lccc} & \text { Nombre } & \text { Nombre } & \text { Fréquence moyenne } \\ \text { d'attestations } & \text { de mots } & \text { d'emploi } \\ \text {-isch } & 135 & 71 & 2 \\ \text {-sch } & 63 & 1 & 63\end{array}$

Il en résulte que la fréquence du mot deutsch (à développement phonétique irrégulier) est beaucoup plus haute que celle des dérivés en -isch (à développement phonétique régulier).

En ce qui concerne le néerlandais, nous avons dépouillé un fragment du journal Leidsch Dagblad du 3 octobre 1989, où nous avons trouvé les formes suivantes en -isch et $-s$ :

-isch: 4 logisch; 3 economisch, historisch; 2 academisch, botanisch, democratisch; 1 automatisch, calvinistisch, dynamisch, Olympisch, strategisch, Tunesisch.

-s: 66 Leids ; 40 Amerikaans; 9 Engels; 6 Nederlands; 4 buitenlands; 3 Duits(land), Hollands; 2 Anglicaans, Japans, westers; 1 aards, Amsterdams, binnenlands, Brits, Europees, Grieks, Hoglands, Londens, Nederduits, protestants, rooms, Spaans, Zeeuws, 16e eeuws, Zuidafrikaans.

Au point de vue statistique, ces données se présentent comme suit:

$\begin{array}{lccc} & \begin{array}{c}\text { Nombre } \\ \text { d'attestations }\end{array} & \begin{array}{c}\text { Nombre } \\ \text { de mots }\end{array} & \begin{array}{c}\text { Fréquence moyenne } \\ \text { d'emploi }\end{array} \\ -i s c h & 22 & 12 & 2 \\ -s & 152 & 25 & 6\end{array}$

Il en est de même dans d'autres langues germaniques (en danois, etc.). Seul l'anglais constitue une exception parce que English, où -ish s'est maintenu tel quel, est plus employé que des dérivés comme Welsh, Scotch, Dutch, French, Scots, Norse ou Manx, où le suffixe a subi une réduction. Cela s'explique par le fait que le groupe de consonnes précédant -ish dans English a empêché la réduction du suffixe.

Notre théorie du développement phonétique irrégulier dû à la fréquence d'emploi a été critiquée par Gougenheim 1970, Guiter 1970, Madonia 1970, Messner 1970, Posner 1970, Butler 1972 et Franceschi 1985. Nous leur avons répondu dans Mańczak 1977: 59-70 et 1986: 383-384. Mais la théorie du développement phonétique irrégulier dû à la fréquence, longtemps considérée comme fausse, commence finalement à être approuvée, voir Lloyd 1987: 34-35, 158, 166 et 360. 


\section{RÉFÉRENCES}

Butler J.L. 1972, c.r. de Mańczak 1969, RomPh (abréviations d'après la Bibliographie linguistique) $25: 331-336$.

Diez F. 1846, Altromanische Sprachderkmale, Bonn.

Franceschi T. 1985, intervention concernant Mańczak 1985.

Gougenheim G. 1970, c.r. de Mańczak 1969, BSL, 65: 58-59.

Guiter H. 1970, c.r. de Mańczak 1969, RLaR, 89: 301-302.

Lloyd P.M. 1987, From Latin to Spanish, Vol. I: Historical Phonology and Morphology of the Spanish Language, Memoirs of the American Philosophical Society, vol. 173.

Madonia G. 1970, c.r. de Mańczak 1969, Phonetica, 22: 189-190.

Mańczak W. 1962, Phonétique et morphologie historiques du français, Lódź.

- 1969, Le développement phonétique des langues romanes et la fréquence, Cracovie.

- 1974, Double issue de oi en français, NphM, 75: 357-367.

- 1977, Le latin classique langue romane commune, Wrocław.

- 1985, Les participes passés du type esp. dial. cantao, it. dial. vestiu et campid. lliberau, in: XVI Congrès Internac. de Ling. i Fil. Romàniques, II, Palma de Mallorca, p. 65-73.

- 1986, Double valeur prosodique de grec $-0 \tau,-\alpha$, BSL, 81: 377-384.

- 1987, Frequenzbedingter unregelmässiger Lautwandel in den germanischen Sprachen, Wroctaw.

Messner D. 1970, c.r. de Mańczak 1969, Die Sprache, 16: 190.

Posner R. 1970, c.r. de Mańczak 1969, Lingua, 25: 440-445.

Schuchardt H. 1885, Über die Lautgesetze. Gegen die Junggrammatiker, Berlin.

Spore P. 1989, Les suffixes -ais et -ois ajoutés à un nom de ville, in: XIX Congreso

Internac. de Ling. e Fil. Románicas. Resumes das comunicacións, Santiago de Compostela, p. 358-359.

Povzetek

ZAKAJ français, PAC PA François?

Lat. -ensem in germ. -isk sta se v francoščini zlila v eno samo pripono, ki pa se pojavlja v dveh oblikah, -ois in -ais. Samo prvo glasovno podobo lahko imamo za fonetično pričakovano, druga, krajša (izg. je è), pa dolguje svojo obliko pogostnosti rabe.

V sinhroniji vlada nekak zakon pogostnosti rabe: močno rabljene glasovne prvine so zmeraj krajše. Vsi jeziki poznajo krajšave, vzemimo fr. chemin de fer métropolitain > métropolitain in še naprej v métro. Prepričljiv primer so tudi razlike med oblikami polnopomenskega in pomožnega glagola: lat. cantare habebatis $>$ chanteriez, ali pa bolj rabljene edninske oblike it. ho, hai, ha proti manj rabljenim množinskim abbiamo, avete.

Težnja h krajšavi lahko pojasni marsikatero fonetično navidezno nepravilnost. Pripona -ais je bila kot značilna pripona za tvorbo etnonimov pogosteje rabljena. 